\title{
The CDF-II Silicon Tracking System
}

\author{
F.Palmonari on behalf of the CDFII Collaboration \\ INFN St.Piero a Grado, via Livornese vecchia 1291, 56100, Pisa, Italy
}

\begin{abstract}
The CDFII silicon tracking system, SVX, for Run II of the Fermilab Tevatron has up to 8 cylindricals layers with average radii spanning from $\sim(1.5$ to 28.7$) \mathrm{cm}$, and lengths ranging from $\sim(90$ to 200$) \mathrm{cm}$ for a total active-area of $\sim 6 \mathrm{~m}^{2}$ and $\sim 7.2 \times 10^{5}$ readout channels. SVX will improve the CDFII acceptance and efficiency for both $\mathrm{B}$ and high-Pt physics dependent upon b-tagging. Along with the description of the SVX we report some alignment survey data from the SVX assembly phase and the actual status of the alignment as it results from the offline data analysis. The problems encountered are also reviewed.
\end{abstract}

\section{Introduction}

The SVX project developed between 1992 and 2001; final assembly started in 1999; on January 16 2001, SVX was installed into CDFII and run IIa started in early march $2001^{1}$. SVX is made of 3 mechanically separate subdetectors L00, SVXII and ISL that exploit different features of the same radiation-hard chip SVX3D for data acquisition[1],[2] (DAQ). The original design of SVXII (4 layers of double sided sensors) was modified to add a fifth layer and the Intermediate Silicon Layers (ISL) and L00 have been added later on (1996 and 1998).

\section{The SVX tracker}

In Figure 1 (right) we show the SVX location in the CDFII detector. The concept of the SVX tracker is that L00 and SVXII provide vertex information at radial co-

\footnotetext{
${ }^{1}$ Tevatron collider is designed to provide collisions at $\sqrt{s}=2.0 \mathrm{TeV}$ center of mass energy with maximum istantaneous luminosity of $2 \times 10^{32} \mathrm{~cm}^{-2} \mathrm{~s}^{-1}$ and interbunch spacing that nowadays is $396 \mathrm{~ns}$ and will be reduced to $132 \mathrm{~ns}$.
} 
ordinates below $\sim 10 \mathrm{~cm}$; L00 inside SVXII (mounted on the beryllium beam-pipe) can enhance charged track's impact parameter $\left(d_{0}\right)$ resolution; SVXII and ISL provide three-dimensional hits $(r, \varphi, z)$; the SVXII ones can feed real-time informations to SVT[3] for trigger purpose while the forward ${ }^{2}$ (backward) ISL are used to track particles where the outer tracking (COT) coverage is not complete. In this way the SVX improves and extends the CDFII tracking capabilities outside COT full coverage limits. Efforts where made to minimize the amount of material. In L00,
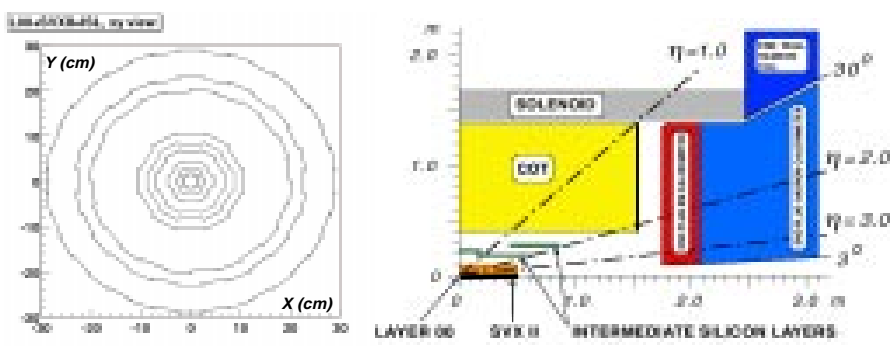

Figure 1: (left) Geometry layout of the SVX=L00+SVXII+ISL silicon layers; (right) Location of SVX inside CDFII for the Run IIa of Tevatron (Fermilab)

Figure 2 (left), lightweight fine-pitch kapton cables $(50 \mu \mathrm{m}$ kapton film, $10 \mu \mathrm{m}$ trace width and maximum length of $47 \mathrm{~cm}$ ) allow all of the front-end electronics (hybrids) to be moved outside the tracking volume. The L00 support structure is made out of a multi-layer layup of high thermal conductivity carbon fiber (CF) with integrated cooling $^{3}$ for the silicon sensors (wafers). The SVXII is arranged into three identical barrels symmetrically mounted with respect to the interaction point. Within each barrel, Figure 2 (center), the low mass constraint is addressed: gluing hybrids on the wafers, integrating cooling channels into the ladder's ${ }^{4}$ beryllium supports (bulkheads) and arranging comunication electronics (portcards) in a external ring. For ISL, Figure 2 (right), the development of two kinds of CF supports (one for each layer) allowed the building of $\sim 50 \mathrm{~cm}$ long modules (i.e. selected[4] pairs of ladders joined together) with hybrids at opposite ends. This minimized the transversed material in the central region. In addition, the $\sim 2 \mathrm{~m}$ long for $\sim 7.3 \mathrm{~kg}$ CF space frame $[5]$ (SF, the supporting structure of ISL), provides the three mount points for the SVXII

\footnotetext{
${ }^{2}$ We will refer to (C) central region where $|\eta| \leq 1$ and to (F/B) forward (or backward) region $1<|\eta| \leq 2$.

${ }^{3}$ The expectation is that L00 wafers can last $\leq 7.4 \mathrm{fb}^{-1}$ if kept at $-5^{\circ} \mathrm{C}$.

${ }^{4}$ Basic SVX active units: 1 wafer in the case of L00, 2 wafers and 2 hybrids glued together and to a CF support for the SVXII and finally 3 wafers and 1 hybrid glued to a common CF support for ISL.
} 
CF-honeycomb space tube (ST) where SVXII is held by 15 CF-glass legs ${ }^{5}$ glued to 3 adjustable mount points (differential screws) per barrel. L00 and the Be beam pipe are clamped to the ST via adjusters and dampers. Externally the SF is closed by a $0.1 \mathrm{~cm}$ thick CF-shell and connected to two CF-honeycomb extension cylinders ( $\sim 9 \mathrm{~kg}$ each after detector cabling) that provide housing for the juction cards (and ISL port-cards). The extension cylinders rest on 6 inchworms (piezo-electric actuators) for the SVX active-alignment. The final weight of SVX, including cabling and cooling, is $\sim 128 \mathrm{~kg}$ and the SF supports more then ten times its weight. In Figure 1 (left) we see the modules layout. Layers are concentric and within each one of them, modules are arranged so that some are closer to the beam line (inner) and others are further from it (outer) ${ }^{6}$. Nevertheless for SVXII, ISL the number of readout channels per ladder within a layer is fixed while, for L00, space constraints led to a variable number (and corresponding use of wide and narrow sensors) (see also Table 1). The resulting geometry has in each layer some overlap regions that not only compensate for the inactive-area of wafer's borders but provide some thin double-hit $\varphi$-sectors (within a layer) that can be used to restore good resolution of tracks intersecting wafers $\varphi$-edges. Several kind of $300 \mu \mathrm{m}$ thick wafers from differ-

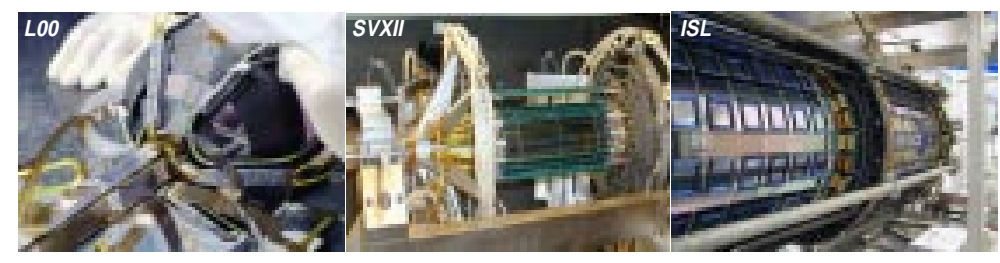

Figure 2: (left) L00 hybrid installation (low-mass cables visible); (center) Module installation under Coordinate Measuring Machine (CMM) during SVXII barrel assembly; (right) Side view of ISL layers 6 central and 7 forward in the "rotation-cage" used for module installation.

ent manufacturers (ST Microelectronics, Micron and Hamamatsu) have been used. All SVX layers are double-sided except for L00. In particular layers 3, 5, 6 and 7 have axial-strips (wafer $r \varphi$ side) on the $p$ side $^{7}$ with small-angle-stereo (SAS) strips (wafer $z$ side; SAS form an angle $|\alpha|=1.2^{\circ}$ with the CDFII longitudinal $z$ axis $^{8}$ on the $n$ side while layers 1,2 and 4 have $n$ strips forming a $90^{\circ}$ angle with $z$ and are capable of accurate longitudinal measurements once the ambiguity due to their

\footnotetext{
${ }^{5}$ With low coefficient of thermal expansion.

${ }^{6}$ Only in ISL: inner modules have wafers $r \varphi$ side facing out of CDFII while outer ones have the $r \varphi$ side facing the beam-line (see below for a definition of the $r \varphi$ side).

${ }^{7}$ Only for layer $6, p$ side is SAS while $n$ side is $r \varphi$.

${ }^{8}$ The CDFII $+z$ versus is defined as the Tevatron proton versus
} 


\begin{tabular}{|c|c|c|c|c|c|c|c|}
\hline $\begin{array}{l}\text { SVX } \\
\text { layer }\end{array}$ & & $\begin{array}{l}m)^{\prime} \\
\text { out }\end{array}$ & $\overline{\text { pitc }}$ & $(\mu \mathrm{m})$ & $\begin{array}{c}\text { stereo } \\
\text { angle } \\
\text { (deg.) }\end{array}$ & $\begin{array}{c} \\
\text { module } \\
\text { [tot. }]\end{array}$ & $\begin{array}{c}\% X_{o} \\
\text { avg. } \\
{[\max .]}\end{array}$ \\
\hline $\begin{array}{l}\mathbf{0} \\
\text { L00 }\end{array}$ & 1.35 & 1.62 & 25 & - & - & $\begin{array}{c}36 / 36 \\
{[72]}\end{array}$ & $0.6[1.1]$ \\
\hline 1 & 2.5 & 3.0 & 60 & 141 & 90 & 36 & \\
\hline 2 & 4.1 & 4.6 & 62 & 125.5 & 90 & 36 & \\
\hline 3 & 6.5 & 7.0 & 60 & 60 & +1.2 & 36 & \\
\hline 4 & 8.2 & 8.7 & 60 & 141 & 90 & 36 & \\
\hline 5 & 10.1 & 10.6 & 65 & 65 & -1.2 & 36 & \\
\hline SVXII & & & & & & [180] & $7.0[15.0]$ \\
\hline $6 \mathrm{C}$ & 22.6 & 23.1 & 112 & 112 & $\mp 1.2$ & 28 & \\
\hline $6 \mathrm{~F} / \mathrm{B}$ & 19.7 & 20.1 & 112 & 112 & \pm 1.2 & 48 & \\
\hline $7 \mathrm{~F} / \mathrm{B}$ & 28.6 & 28.9 & 112 & 112 & $\mp 1.2$ & 72 & \\
\hline ISL & & & & & & [148] & $1.0[5.0]$ \\
\hline
\end{tabular}

Table 1: Summary of the SVX layout. In the last column we report an estimate for the average (and maximum) $\% X_{o}$ (for $90^{\circ}$ incidence) transversed in each SVX subdetector[6]. Maximum values refer to $(r, \varphi)$ regions where hybrids, cooling pipes, etc. are. For L00: when two values are reported they refer to narrow or wide number of wafers and while the strip pitch is reported the readout pitch is $50 \mu \mathrm{m}$.

readout ganging (multiplexing) is resolved. In Table 1 we summarize some SVX quantities.

\section{$2.1 \quad$ SVX cooling}

Some evaluated quantities of the SVX cooling system [7], [8] are in Table 2. Referring to the blocked ISL cooling lines[4], on November 22001 a thin layer of glue was removed on the $6 \mathrm{C}$ layer using a laser technique[9]. Full efficiency was recovered for the region $\left(22.6 \mathrm{~cm}<r<23.1 \mathrm{~cm} ; 77^{\circ}<\varphi<180^{\circ} ;-0.75<\eta<0.0\right)$. By employing this technique during future accesses to the detector, we expect to recover the $\simeq 33 \%$ of it that is tested and ready for DAQ so to restore full ISL capabilities. 


\begin{tabular}{|l|c|c|c|c|}
\hline (hybrid cooling only) & L00 & SVXII & ISL & totals \\
\hline power to dissipate (KW) & 0.6 & 1.4 & 1.2 & 2.7 \\
length of cooling circuits $(\mathrm{m})$ & 8 & 52 & 38 & 98 \\
$\mathrm{H}_{2}$ O to fill circuits $(\mathrm{l})$ & 0.81 & 1.35 & 1.78 & 3.94 \\
(cold mass)/(hot mass) & 1.20 & 0.57 & 0.75 & \\
\hline
\end{tabular}

Table 2: Evaluated quantities of the SVX hybrid cooling system. All lengths are taken into account starting from COT faces. The hot, cold masses are computed taking into account only the ones that undergo heat exchanges.

\section{$3 \quad$ SVX alignment}

The SVXII stand-alone was designed[10] to resolve track's impact parameters with $\sigma_{d_{0}}<50 \oplus 80 /\left(p_{T} \sqrt{\sin (\theta)}\right)$ and transverse momentum with $\delta p_{T} / p_{T}^{2} \sim 7 \%(\mathrm{GeV} / c)^{-1}$. Since the L00 and ISL addition, these expected resolutions are $\sigma_{d_{0}}<15 \oplus 25 /\left(p_{T} \sqrt{\sin (\theta)}\right)$ and $\delta p_{T} / p_{T}^{2} \sim 0.4 \%(\mathrm{GeV} / c)^{-1}$ in fact (already in[10]) we rely on the capability to develop alghorithms for pattern recognition optimization that correctly pick and collect hits belonging to a track through all sub-detectors. Increasing track purity and $p_{T}$ resolution using the lever arm of ISL and enhancing $\sigma_{d_{0}}$ with L00 are achievable once all the corrections of the final-alignment will be applied. This procedure began, for example, in[11]. However, these expected results rely on the assembly-alignment quality and stability.

\subsection{Stability}

The ISL space frame stability was evaluated over a period of 9 months measuring maximum radial variations $<44 \mu \mathrm{m}$. Also SF and ST deformations under full load were addressed with deflection tests measuring maximum displacements $<55 \mu \mathrm{m}$ in both. A step further in the stability survey of sub-detectors relative alignment was the installation of a RASNIK network[12] (CCD cameras). This will keep an on-line and historical trace of L00-SVXII and ISL-COT relative movements.

\subsection{Survey data}

Before assembly, efforts were made to minimize and characterize module deflection and deformation. Deflections at $\varphi=\frac{\pi}{2}, \frac{3}{2} \pi$ are $<65 \mu \mathrm{m}$ for SVXII and $<150 \mu \mathrm{m}$ 
for even ledges ${ }^{9}$ or less then $120 \mu \mathrm{m}$ for odd ledges in ISL, depending on module orientation $^{10}$. Deformations due to thermal stresses are a concern. The option to clamp firmly only one of the modules ends was chosen for both SVXII and ISL. Let $\delta \alpha$ be the angle to which wafers strips are not parrallel to the CDFII $+z$ axis. In SVXII and ISL ${ }^{11}$ after ladder alignment and assembly (656 ladders, with lengths up to $27.82 \mathrm{~cm}$ ), wafer strips deflect from an ideal line by $\delta \alpha<10.0 \mu \mathrm{rad}$. Module mounting onto bulkheads and ledges or wafers gluing onto CF structure introduced further deviations. Measured numbers for L00 and SVXII once completed are $\delta \alpha_{l 00}<26 \mu \mathrm{rad}$ and $\delta \alpha_{\text {svxii }}<34 \mu \mathrm{rad}$. In addition, the SVXII barrel center $(r, \varphi)$ offsets were $(<40 \mu \mathrm{m},<2.1 \mathrm{mrad})$ and slope differences contained in less then $70 \mu \mathrm{rad}$. For ISL we have only an indirect evaluation. Assuming that modules clamped onto SF ledges inherit the direction defined by their mating-ledge slots then $\delta \alpha_{i s l}<220 \mu \mathrm{rad}$. The average axis used for the three sub-detectors alignment, re-mesured once SVX was completed, have $\delta \alpha_{s v x}<150 \mu \mathrm{rad}$ (SF length is 197.16 $\mathrm{cm})$; in this reference frame, the maximum recorded discrepancy from a nominal value was $300 \mu \mathrm{m}$. All these quantities are summarized in Table 3.

\subsection{Collision data}

Preliminary results return unbiased residual distributions with $\sigma<13 \mu \mathrm{m}$ (for all SVXII layers in each barrel) and barrel-to-barrel differences in slopes within $<200 \mu \mathrm{rad}$. We are finding efficient track patterns with attached ISL hits and we have indication that SVXII-ISL layers are parallel within less then $178 \mu \mathrm{rad}$.

\section{Conclusions}

A description of the SVX, the silicon tracking system of CDFII, and some details related to its internal alignment were reported. SVX will become "tracker" only once the necessary software that efficently performs SVX-COT track linking is optimized. The material budget in the tracking volume is worse than expected but the good quality of the overall SVX assembly is already allowing fast progress of the alignment corrections study. We also welcome the recent news proving that the major concern, the ISL cooling, is on its way to being fixed.

\footnotetext{
${ }^{9} 5.0 \mathrm{~cm} \times 1.45 \mathrm{~cm} \times 0.1 \mathrm{~cm}$ beryllium plates where ISL modules are clamped.

${ }^{10}$ Ledge $n=0$ is at $\varphi=0$ in layer $6 \mathrm{C}, 7 \mathrm{~F} / \mathrm{B}$ and $\varphi=-7.5^{\circ}$ in $6 \mathrm{~F} / \mathrm{B} ; n$ increase anti-clockwise.

${ }^{11}$ As pointed out, we cannot really speak of ladders for L00. However the following limit $\delta \alpha_{l 00}$ is valid for three longitudinally subsequent L00 wafers.
} 


\begin{tabular}{|c|c|}
\hline \multicolumn{2}{|c|}{ Survey before assembly } \\
\hline Ladders internal alignment (SVXII,ISL) & $\delta \alpha<10 \mu \mathrm{rad}$ \\
\hline ISL module $\sim 50 \mathrm{~cm}$ long C.C.O. [odd] & $<170 \mu \mathrm{m}$ \\
\hline [even] & $<145 \mu \mathrm{m}$ \\
\hline SVXII module $\sim 30 \mathrm{~cm}$ long C.C. & $<65 \mu \mathrm{m}$ \\
\hline Stability (SF, ST under full load) & $<55 \mu \mathrm{m}$ \\
\hline \multicolumn{2}{|c|}{ Survey after assembly } \\
\hline $\begin{array}{l}\text { Modules alignment } \\
(*) \\
\text { SVXII barrels centers }(\mathrm{r}, \varphi) \text { offsets } \\
\text { SVXII barrels slopes }\end{array}$ & $\begin{array}{c}\delta \alpha_{l 00}<26 \mu \mathrm{rad} \\
\delta \alpha_{\text {svxii }}<34 \mu \mathrm{rad} \\
\delta \alpha_{i s l}<220 \mu \mathrm{rad} \\
(<40 \mu \mathrm{m},<2.1 \mathrm{mrad}) \\
<70 \mu \mathrm{rad}\end{array}$ \\
\hline \multicolumn{2}{|c|}{ Preliminary results from collision data } \\
\hline Modules alignment & $\begin{array}{l}\delta \alpha_{\text {svxii }}<34 \mu \mathrm{rad} \\
\delta \alpha_{i s l}<178 \mu \mathrm{rad}\end{array}$ \\
\hline
\end{tabular}

Table 3: SVX alignment summary. The reported values are upper limits. By C.C. we mean that clamping and cooling deformations are considered; by C.C.O. that also orientation of modules into $\mathrm{SF}$ are considered. Flags [odd]/[even] refer to ISL ledges. The $\left(^{*}\right)$ indicate an evaluation and not a direct measure. 


\section{References}

[1] M.Bishai for the CDF coll., The silicon data acquisition system and front-end electronics for CDF Run II, These Proceedings.

[2] S.D'Auria for the CDF coll., Commissioning and Operation of the CDF SVX detector, These Proceedings.

[3] I.Fiori for the CDF coll., CDF online Silicon Vertex Tracker, These Proceedings.

[4] A.Affolder et al., Status report of the ISL detector at CDFII, Proceedings 5th Int. Conf. Large Scale Applications and Radiation Hardness of Semiconductor Detectors, Firenze, Italy, 2001.

[5] A.Basti et al., The Intermediate Silicon Layers Space Frame, Proceedings 4th Int. Conf. Large Scale Applications and Radiation Hardness of Semiconductor Detectors, Firenze, Italy, 1999.

[6] D.Stuart, CDF note 5268, 2000.

[7] S.Blusk et al., Characterization of a SVXII Bulkhead, CDF note 4473, 1998.

[8] A.Basti et al., The Intermediate Silicon Layers Cooling System, CDF note 5034, 1999.

[9] D.Glenzinski (CDF coll.) private communication.

[10] CDFII coll., The CDFII detector Technical Design Report, Fermilab-Pub. 390-E, 1996.

[11] R.McNulty, T.Shears, A.Skiba A procedure for the software alignment of the CDF Silicon System, CDF note 5700, 2001.

[12] E.Sanders, D.Saltzberg Proposal for a Rasnik Relative Alignment Monitor for the CDFII SVX-ISL-COT, CDF internal note 4510, February 1998. 